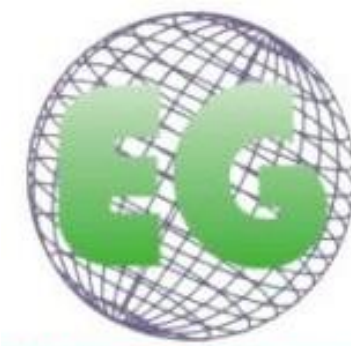

ISSN 1695-6141 $N^{\circ} 56$
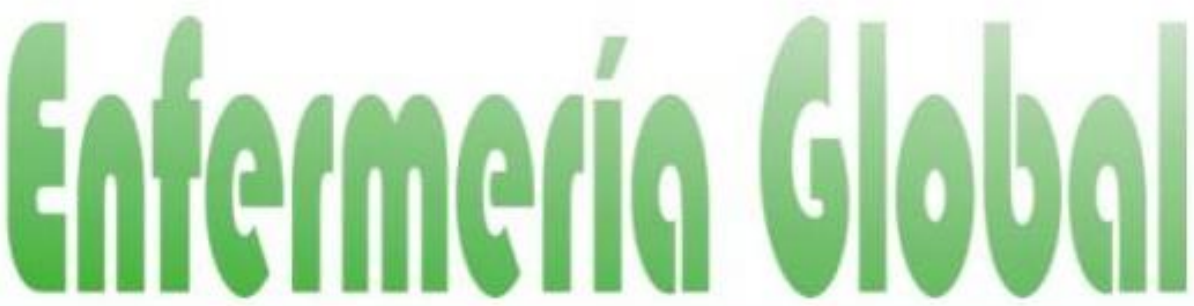

Revista electrónica trimestral de Enfermería

Octubre 2019

www.um.es/eglobal/

ORIGINALES

\title{
Conocimiento de adultos jóvenes sobre \\ cerebrovascular en una ciudad del sur de Portugal \\ el accidente
}

Conhecimento de adultos jovens sobre o acidente vascular cerebral numa cidade ao sul de Portugal

Young adult's knowledge about stroke in a Portuguese south town

Maria Margarida Santana Fialho Sim-Sim ${ }^{1}$

Maria José Abrantes ${ }^{1}$

Maria Gorete Mendonça dos Reis ${ }^{1}$

Elsa Maria Garção Pires ${ }^{2}$

Manuel Agostinho Matos Fernandes ${ }^{1}$

Maria da Luz Ferreira Barros ${ }^{1}$

${ }^{1}$ Universidad de Évora. Portugal. msimsim@uevora.pt

${ }^{2}$ Hospital del Espíritu Santo EPE de Évora. Portugal.

\section{http://dx.doi.org/10.6018/eglobal.18.4.356061}

Recibido: 28/12/2018

Aceptado: $15 / 02 / 2019$

\section{RESUMEN:}

Objetivo: Analizar el conocimiento de adultos jóvenes sobre el accidente cerebrovascular.

Métodos: Estudio cuantitativo, transversal, en ambiente no-clínico. Muestra de conveniencia de adultos. A través de los niños de las escuelas de la ciudad de Évora, se pidió la participación de las figuras parentales. Los aspectos éticos fueron respetados. Cuestionario de auto rellenado devuelto pasado una semana.

Resultados: Participaron 147 hombres (44.5\%), 183 mujeres (55.5\%), con la edad media de 42.25 años $(\mathrm{DP}=5.00)$. El comportamiento de riesgo más referido para el accidente cerebrovascular es el tabaquismo (56.5\%), siguiéndo la hipertensión (28.8\%) y la hipercolesterolemia (28.8\%). Las principales fuentes de información son la televisión (93,2\%) y los amigos (89,4\%). En la ayuda inmediata, las actitudes de los participantes serían en la mayoría incorrectas, existiendo un desfase entre el conocimiento y la respectiva actuación.

Conclusiones: Existen entre los participantes comportamientos de riesgo observados en otros estudios con población de personas mayores. Los resultados detectan déficit de alfabetización en salud. Será urgente el desarrollo de proyectos que informen y ejemplifiquen, los riesgos de los comportamientos. La actuación ante la persona con accidente cerebrovascular, debe ser objeto de enseñanza y de entrenamiento al ciudadano. La divulgación de información sobre accidente cerebrovascular es urgente en la región. Los adultos jóvenes, como educadores, pueden intervenir para disminuir los casos en la generación de sus hijos.

Palabras Clave: conocimiento; accidente cerebrovascular; factores de riesgo.

\section{RESUMO:}

Objetivo: Analisar o conhecimento de jovens adultos sobre o AVC. 
Método: Estudo quantitativo, transversal, em ambiente não clínico. Amostra de conveniência de adultos. Através das crianças das escolas da cidade de Évora, foi solicitada a participação das figuras parentais. Os aspetos éticos foram respeitados. Questionário de auto-preenchimento com retorno após uma semana.

Resultados: Participaram 147 homens (44,5\%), 183 mulheres (55,5\%), com idade média de 42,25 anos $(\mathrm{DP}=5,00)$. O comportamento de risco mais referido ao acidente vascular cerebral é o tabagismo (56,5\%), seguido da hipertensão $(28,8 \%)$ e hipercolesterolemia $(28,8 \%)$. As principais fontes de informação são televisão (93,2\%) e amigos $(89,4 \%)$. Na ajuda imediata das vitimas, as atitudes dos participantes seriam em sua maioria incorretas, existindo uma lacuna entre o conhecimento e 0 respetivo desempenho.

Conclusões: Existem comportamentos de risco entre os participantes, que são observados em outros estudos, mas com população de idosos. Os resultados detetam carência na literacia em saúde. Será urgente o desenvolvimento de projetos que informem e exemplifiquem os riscos dos comportamentos. A ação perante a pessoa vítima de acidente vascular cerebral, deve ser objeto de educação e treino do cidadão. A informação sobre a temática é urgente na região. Jovens adultos, como educadores, pode intervir para reduzir os casos na geração dos filhos.

Palavras-chave: conhecimento; acidente vascular cerebral, fatores de risco.

\section{ABSTRACT:}

Aim: To analyze the knowledge of young adults about stroke.

Method: Quantitative, cross-sectional study in a non-clinical environment. Convenience simple. Through the elementary school of Evora, parental figures were asked to participate. Ethical aspects were respected. Self-fill questionnaire returned after one week.

Results: 147 men (44.5\%) participated, 183 women (55.5\%), with a mean age of 42.25 years (SD = 5.00). The most commonly reported risk behavior for stroke is smoking (56.5\%), followed by hypertension (28.8\%) and hypercholesterolemia (28.8\%). The main sources of information are television $(93.2 \%)$ and friends (89.4\%). In immediate witness assistance, the attitudes of the participants would be mostly incorrect. There is a gap between knowledge and the respective action.

Conclusions: there is a risk behavior, observed also among other studies, with a population of older people. The results show a lack of literacy in health. It will be urgent to develop projects that inform and exemplify the risks of behavior. The performance of the person with stroke must be taught and trained to the citizen. The dissemination of information about stroke is urgent in the region. Young adults, as educators, can intervene to reduce cases in the generation of their children.

Keywords: knowledge; stroke; risk factors; Attitude.

\section{INTRODUCCIÓN}

Las enfermedades cerebrovasculares son disfunciones que afectan temporal o permanentemente el flujo sanguíneo. Contribuyen a la mortalidad en el mundo, siendo esta enfermedad, el accidente cerebrovascular (ACV), la segunda causa de muerte desde hace 16 años ${ }^{(1)}$. Hace décadas y en diferentes lugares, las enfermeras consideran esta patología un tema importante para investigación ${ }^{(2)}$. Los factores de riesgo no modificables se refieren a la progresión de la edad o al patrimonio genético. El ACV contextualizado en la edad, tiene expresiones variadas en los perjuicios inmediatos y futuros para la persona. Es referido como poco común en adultos jóvenes (i.e., 10-15\%) registrando todavía en el rango etario 45-49 años diferencias de incidencia que varían entre $5 \%$ y $20 \%{ }^{(3)}$. No obstante, en adultos jóvenes la mortalidad es elevada y la morbilidad es acompañada de angustia, debido a las secuelas con las que se enfrentan en la fase más productiva de la vida ${ }^{(3-5)}$. En las mujeres, los factores específicos asociados al embarazo, puerperio, al uso de anticonceptivos o pre-climaterio, incrementan el riesgo ${ }^{(5,}$, 6). Considerándose la genética ubicada en factores étnicos, los riesgos más grandes se encuentran en las mujeres negras no hispánicas versus riesgos menores en las caucasianas ${ }^{(6)}$. Si hasta los 35-44 años los individuos del sexo masculino presentan una mayor prevalencia, alrededor del rango 45-54 años, se vuelve superior en las mujeres ${ }^{(4)}$. 
Los factores modificables, por su lado, se manifiestan de múltiples formas en las conductas no saludables, que traducen el nivel de educación ${ }^{(6)}$. Se destacan la hipertensión, diabetes, tabaquismo, obesidad, dislipidemias, inactividad. Otros comportamientos abusivos como el del alcohol, la sal, el azúcar, pueden potencializar los anteriores ${ }^{(5,7,8)}$. Otros aspectos, tales como nivel o la posición socioeconómica, influyen sobre algunos factores modificables, mostrando una asociación inversa con la incidencia y mortalidad del ACV ${ }^{(9,10)}$.

En Portugal el ACV también es la segunda causa de muerte. Pero en el Sur, en el Alentejo, la situación es más grave, tanto en la mortalidad (57.2/100.000 hab), como el la morbilidad ${ }^{(11)}$. En el Alentejo, el tabaquismo muestra valores más elevados, en comparación con los datos del país, en cualquier grupo etario ${ }^{(12)}$. En cuanto al exceso de peso, éste es menos frecuente en comparación con otras regiones, pero la prevalencia de la HTA y la diabetes es la más elevada del país ${ }^{(13-16)}$.

El reconocimiento inmediato del $\mathrm{ACV}$, la precocidad del auxilio y el direccionamiento de la persona para una unidad de salud, en un espacio de tiempo de hasta 3 horas, puede ofrecer un desenlace diferente al problema. En Portugal la Dirección General de la Salud (DGS), considera relevante la educación para la salud, donde se incluye el reconocimiento por parte de la población de las señales de alarma de las situaciones amenazadoras, así como la activación Vía Verde ACV pre-hospitalaria. La Via Verde ACV es una estrategia organizada para la atención de pacientes con sospecha de padecer ACV en curso. Está reglamentada por la DGS. La Vía Verde ACV es activada por el número de emergencia sanitaria (112) que envía los medios y traslada a la persona al hospital más próximo que cuente con una Unidad de ACV, ya que la atención precoz puede revertir un ACV en curso. Aparte de los recursos relacionados con los servicios de salud y la atención inmediata por los testigos, es fundamental el reconocimiento por parte del paciente de los signos o síntomas del evento. El conocimiento sobre tal cuestión puede ser adquirido a través de programas educativos. Algunos estudios muestran que, en los grupos de riesgo, la información aportada permanece cerca de cinco años ${ }^{(17)}$. Los programas centrados en los grupos específicos son más eficaces. El diseño depende de los factores de riesgo, el contexto y de la propia metodología.

En el Alentejo no se ha estudiado el conocimiento sobre el ACV en adultos jóvenes. No existe una referencia estándar a partir de la cual se pueda tener un entendimiento global de la preparación de los ciudadanos para evitar o intervenir en una situación de ACV. Tampoco las necesidades de información están identificadas. Si existiera conocimiento se crearían condiciones para tomar las decisiones más adecuadas en un espacio de tiempo corto. Considerando el lugar que los adultos jóvenes ocupan entre las generaciones de sus padres y de sus hijos, se realiza el estudio actual, que mira a analizar sus conocimientos sobre las señales y síntomas, así como la rapidez de auxilio ante un episodio de ACV.

\section{METODOLOGÍA}

Estudio cuantitativo, descriptivo transversal. Los participantes son un muestreo de ciudadanos adultos. La captación de la muestra se obtuvo a partir de los padres/madres de niños que están escolarizados en la enseñanza básica. Los criterios de inclusión propuestos fueron: vivir en la ciudad de Évora, tener menos de 
60 años. Se excluyeron las figuras parentales que no viven en la misma casa que el niño.

La selección recayó sobre este grupo, dado que los padres se encuentran en la franja de los adultos jóvenes y simultáneamente conviven en la familia 3 generaciones (hijos, padres, abuelos) De un total de 607 niños, se estimó 1214 figuras parentales. Aplicándose el criterio de Krejce y Morgan ${ }^{(18)}$, se estimó 300 sujetos. Para compensar pérdidas se entregó un $20 \%$ más de cuestionarios, un total de 360 . Se devolvieron totalmente rellenados, 338. Se retiraron del estudio ocho abuelos que son responsables de educación. El estudio recae sobre 330 participantes.

En la encuesta había cuatro secciones: a) variables sociodemográficas (edad, sexo, nivel de educación, b) variables de conocimientos sobre el ACV (i.e., señales, síntomas, condiciones y patologías asociadas), c) variables de riesgo de ACV reconocidos por los participantes (i.e., diabetes, hipertensión, obesidad, hipercolesterolemia, enfermedad cardíaca, tabaquismo) y d) variables de las señales de instalación de ACV (i.e., pictograma Cincinnati) y opciones de auxilio ante una persona con sospecha de ACV.

A partir de las variables de conocimientos del riesgo, de la lista de posibles enfermedades desencadenantes y de las señales de instalación, se constituyó la variable principal: Conocimientos sobre ACV, basado en estudios anteriores ${ }^{(19,20)}$. La puntuación de la variable principal variaba de 0 a 30 puntos. Se obtuvo a través de la suma, considerando en las 30 preguntas las afirmaciones correctas.

Para el análisis de datos se utilizó el IBM-SPSS ${ }^{\circledR}$, versión 22. Para caracterización del muestreo se utilizó la estadística descriptiva. Debido a la no normalidad de la distribución en la variable principal $(\mathrm{K}-\mathrm{S}=.123$; $\mathrm{df}=330 ; \mathrm{p}=.000)$ son utilizadas pruebas no-paramétricas.

El proyecto fue enviado al Centro de Investigación en Ciencias y Tecnologías de la Salud de la Universidad de Évora (CICTS), obteniendo un parecer positivo de la Comisión de Ética de la Universidad (i.e., registro nㄴ4278; parecer nํ15043). Junto de la Dirección Regional de Educación del Alentejo se obtuvo permiso para acceder a las escuelas. Se solicitó la colaboración de los profesores para contactar con las figuras parentales y para la entrega y recogida de los cuestionarios. El cuestionario se entregó, en sobre opaco y cerrado, a los participantes y se solicitó el respectivo consentimiento escrito, con garantía de anonimato y confidencialidad. A las dos semanas todos los cuestionarios estaban cumplimentados

\section{RESULTADOS}

Participaran 147 hombres (44.5\%), 183 mujeres (55.5\%), con edades comprendidas entre 30 y 55 años $(M=42.25$; $D P=5.00)$, sin embargo 15 personas no escribieron la edad. Considerando los 170 participantes que reconocen, en su propia persona, factores de riesgo, a través de análisis de respuestas múltiples, se observa que hay 243 referencias a factores de riesgo. El tabaquismo, la hipertensión y la hipercolesterolemia son los más referidos (tabla 1). 
Tabla 1 - Reconocimiento en sí mismo de prácticas o condición orgánica de riesgos para ACV

\begin{tabular}{llll}
\hline & \multicolumn{3}{l}{ Respuestas } \\
& N & Porcentaje & Porcentaje de Casos \\
\hline Diabetes & 8 & $3,3 \%$ & $4,7 \%$ \\
HTA & 49 & $20,2 \%$ & $28,8 \%$ \\
Obesidad & 34 & $14,0 \%$ & $20,0 \%$ \\
Hipercolesterolémia & 49 & $20,2 \%$ & $28,8 \%$ \\
Enfermedad Cardíaca & 7 & $2,9 \%$ & $4,1 \%$ \\
Tabaquismo & 96 & $39,5 \%$ & $56,5 \%$ \\
Total & 243 & $100,0 \%$ & $142,9 \%$ \\
\hline
\end{tabular}

Analizando separadamente hombres y mujeres, se observa que el tabaquismo es el más referido por los dos sexos. En segundo lugar, el mayor riesgo percibido para los hombres es la hipertensión (32 veces expresado, $25.4 \%$ del sexo masculino), sin embargo, para las mujeres es la hipercolesterolemia (32 veces expresado; $27.4 \%$ del sexo femenino).

En los 96 fumadores, el consumo diario varía entre 2 y 40 cigarros, con una moda de $10(n=22 ; 23.4 \%)$. Relativo a la hipertensión, son 41 (12.9\%) los participantes que toman terapéutica, teniendo edades comprendidas entre los 31 y 50 años $(M=44.38$; $\mathrm{DP}=4.28)$.

La casi totalidad de los participantes ya oyeron hablar de los ACV ( $n=324 ; 99.1 \%)$. Las fuentes donde obtuvieron la información están referidas en la tabla 2, destacándose la televisión y los amigos. La familia es la segunda menor fuente de información.

Tabla 2 - Fuentes de información sobre la temática del AVC

\begin{tabular}{lll}
\hline & Sí (\%) & No \% \\
\hline Televisión & $302(93.2 \%)$ & $22(6.8)$ \\
Amigos & $277(89.4 \%)$ & $33(10.6 \%)$ \\
Escuela & $145(49.8 \%)$ & $146(50.2 \%)$ \\
Consulta/Hospital & $191(64.3 \%)$ & $106(35.7 \%)$ \\
Família & $161(53.5 \%)$ & $140(46.5 \%)$ \\
\hline
\end{tabular}

Para la mayoría de las personas ( $n=309 ; 94,5 \%)$ el ACV se puede prevenir, pero no se puede curar $(n=189 ; 58.3 \%)$. La mayor parte de los participantes consideran que los servicios de salud informan poco sobre el ACV ( $n=207 ; 64.7 \%)$.

La variable principal expresa un nivel de conocimientos sobre el ACV, que varía entre los 16 y 30 puntos, con media de $23.55(\mathrm{DP}=2,58)$. No se observa ninguna diferencia significativa entre los hombres y las mujeres $\left(U=13522.50 ; N_{\text {Mas }}=147 ; N_{\text {Fem }}=183\right.$; $\mathrm{p}=.933)$.

En lo que respecta al conocimiento sobre el ACV, concretamente en lo relativo a la posición en la que debe ser colocado el paciente (sentada, puesta de lado, no sabía que hacer), se verifica, en la comparación por pares, que los participantes que lo colocarían de lado poseen un nivel de conocimiento más elevado. Esto es, a través 
de un test Kruskall-Wallis, se verifican diferencias significativas en los conocimientos, en por lo menos, uno de los grupos $\left(\mathrm{H}_{(2)}=8.406 ; \mathrm{p}=.015\right)$. En las múltiples comparaciones de las ordenes de Dunn se constata que los participantes que no sabían lo que hacer, tenían puntuaciones significativamente más bajas comparativamente a los participantes que acostaban a la víctima en posición lateral o que la sentaban. Sin embargo, por término medio, la ordenación de los conocimientos de aquellos que sentarían a la víctima es superior comparativamente a aquellos que elegirían por el decúbito lateral (figura 1).

Figura 1 Comparaciones múltiples de las órdenes de Dunn para la variable de los conocimientos de los participantes en función de la resolución para la postura de la víctima

\section{Comparaciones entre parejas de C19Posición}

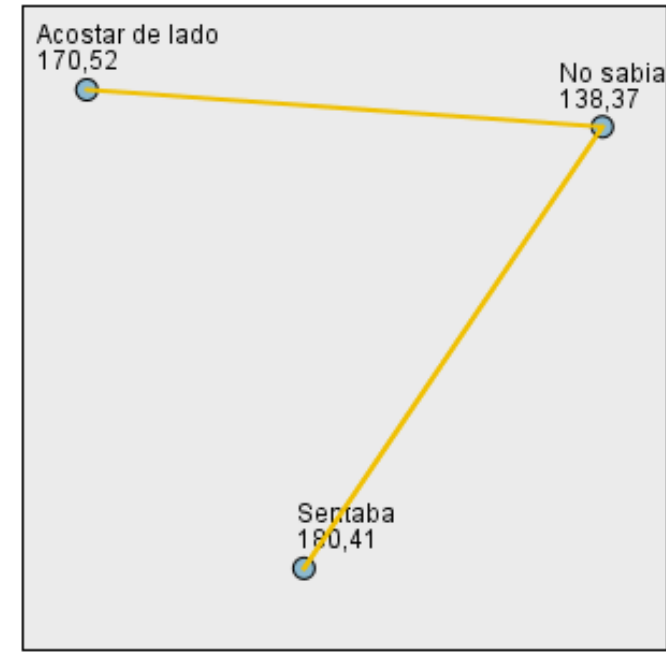

Cada nodo muestra el rango promedio de muestras de C19Posición.

\begin{tabular}{|lrrrrr|}
\hline Muestra 1-Muestra 2 & $\begin{array}{c}\text { Estadístico } \\
\text { de contraste }\end{array}$ & $\begin{array}{c}\text { Error } \\
\text { estándar }\end{array}$ & $\begin{array}{c}\text { Desv. Estadístico } \\
\text { de contraste }\end{array}$ & Sig. & Sig. ajust. \\
\cline { 2 - 7 } No sabia-Acostar de lado & 32,150 & 13,039 & 2,466 &, 014 &, 041 \\
\hline No sabia-Sentaba & 42,039 & 15,613 & 2,693 &, 007 &, 021 \\
\hline Acostar de lado-Sentaba & $-9,889$ & 13,102 &,- 755 &, 450 & 1,000 \\
\hline
\end{tabular}

Cada fila prueba la hipótesis nula de que las distribuciones de la Muestra 1 y la Muestra 2 son las mismas.

Se muestran las significaciones asintóticas (pruebas bilaterales). El nivel de significación es de ,05. Los valores de significación se han ajustado con la corrección Bonferroni en varias pruebas.

Realizándose el análisis de los conocimientos en el cuidado relacionado con la respiración, se constata a través del test Kruskall-Wallis que existen diferencias significativas $\left(\mathrm{H}_{(2)}=11.287 ; \mathrm{p}=.004\right)$. En las múltiples comparaciones de las ordenes de Dunn, los participantes que no sabían lo que hacer, presentan un score de conocimiento significativamente más bajo que aquellos que mandaban toser (figura 2). 
Figura 2: Comparaciones múltiples de las órdenes de Dunn para la variable de los conocimientos de los participantes en función de la resolución para la ventilación de la víctima

\section{Comparaciones entre parejas de C19Respiración}

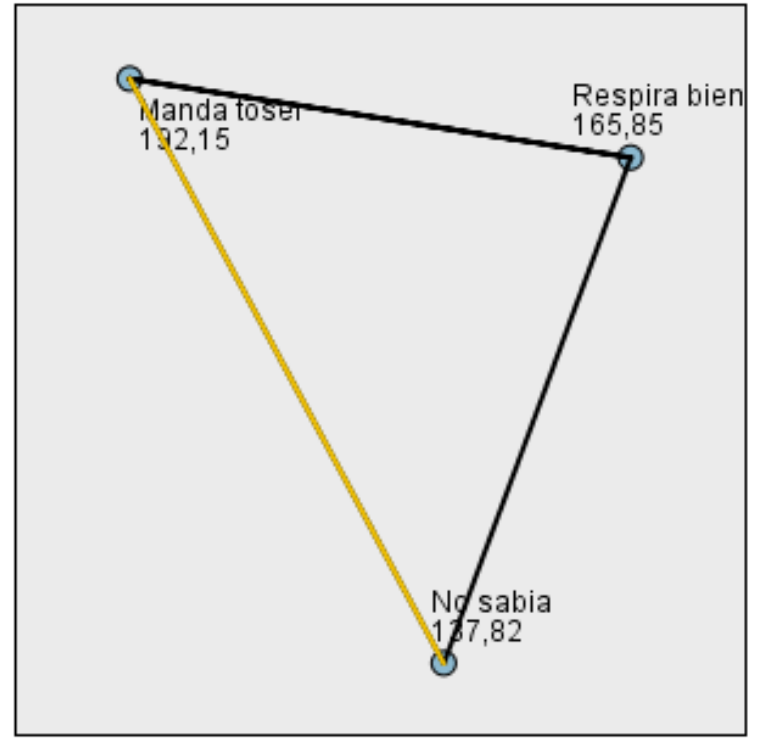

Cada nodo muestra el rango promedio de muestras de C19Respiración.

\begin{tabular}{|lrrrrr|}
\hline Muestra 1-Muestra 2 & $\begin{array}{c}\text { Estadístico } \\
\text { de contraste }\end{array}$ & $\begin{array}{c}\text { Error } \\
\text { estándar }\end{array}$ & $\begin{array}{c}\text { Desv. Estadístico } \\
\text { de contraste }\end{array}$ & Sig. & Sig. ajust. \\
\cline { 2 - 7 } No sabia-Respira bien & 28,028 & 13,098 & 2,140 &, 032 &, 097 \\
\hline No sabia-Manda toser & 54,330 & 16,199 & 3,354 &, 001 &, 002 \\
\hline Respira bien-Manda toser & $-26,302$ & 13,533 & $-1,944$ &, 052 &, 156 \\
\hline
\end{tabular}

Cada fila prueba la hipótesis nula de que las distribuciones de la Muestra 1 y la Muestra 2 son las mismas.

Se muestran las significaciones asintóticas (pruebas bilaterales). El nivel de significación es de ,05. Los valores de significación se han ajustado con la corrección Bonferroni en varias pruebas.

A través de un test Kruskal-Wallis para muestras independientes, se observó que existían diferencias significativas en relación al nivel de conocimiento de los participantes $\left(H_{(2)}=10.378 ; p=.006\right)$ cuando se consideran, los tres grupos de opciones (i.e., llamar al número de emergencia, llamar al 115, no saber qué hacer). De hecho, son las personas que llamarían al 115 (antiguo número de urgencias), aquellas que tienen conocimientos más elevados En las múltiples comparaciones de las ordenes de Dunn, se constata que los participantes que no sabían lo que hacer, presentan un score de conocimientos significativamente más bajo comparativamente a los grupos que llamarían para al 115 o al 112. Sin embargo, el término medio de ordenaciones de los conocimientos de los participantes que llamarían al 112 es más bajo comparativamente a aquellos que llamarían al 115 (figura 3). 
Figura 3 Comparaciones múltiples de las órdenes de Dunn para la variable de los conocimientos de los participantes en función de la resolución para llamar al número de emergencia

\section{Comparaciones entre parejas de C19Recurso}

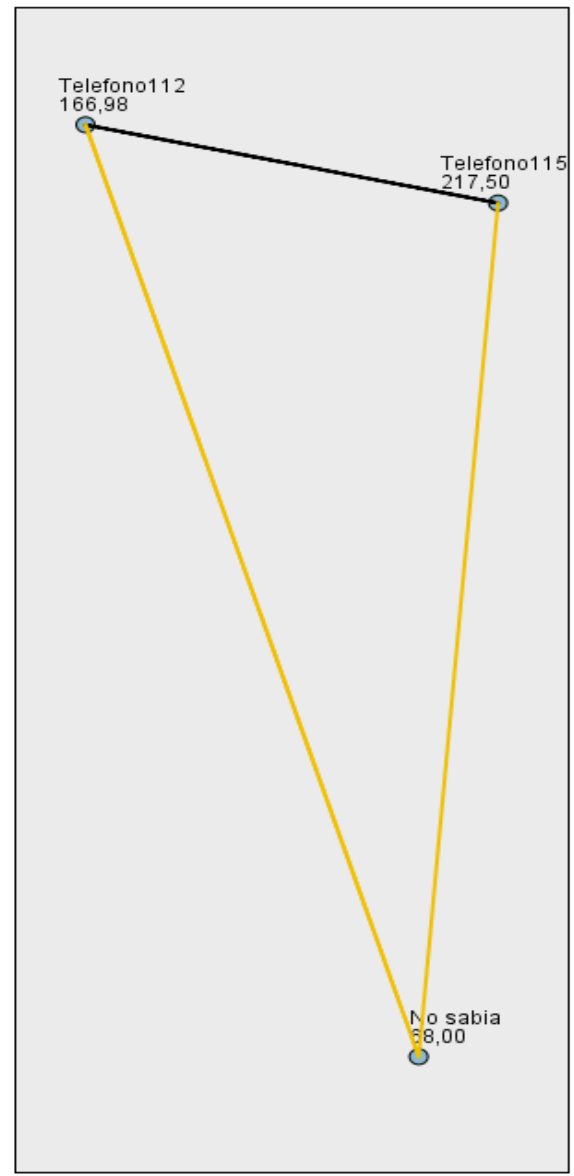

Cada nodo muestra el rango promedio de muestras de C19Recurso.

\begin{tabular}{|lrrrrrr|}
\hline Muestra 1-Muestra 2 & $\begin{array}{c}\text { Estadístico } \\
\text { de contraste }\end{array}$ & $\begin{array}{c}\text { Error } \\
\text { estándar }\end{array}$ & $\begin{array}{c}\text { Desv. Estadístico } \\
\text { de contraste }\end{array}$ & Sig. & Sig. ajust. \\
\cline { 2 - 7 } No sabia-Telefono112 & 98,981 & 33,884 & 2,921 &, 003 &, 010 \\
\hline No sabia-Telefono115 & 149,500 & 51,116 & 2,925 &, 003 &, 010 \\
\hline Telefono112-Telefono115 & 50,519 & 39,005 & 1,295 &, 195 &, 586 \\
\hline
\end{tabular}

Cada fila prueba la hipótesis nula de que las distribuciones de la Muestra 1 y la Muestra 2 son las Se muestran las significaciones asintóticas (pruebas bilaterales). El nivel de significación es de ,05.
Los valores de significación se han ajustado con la corrección Bonferroni en varias pruebas.

\section{DISCUSIÓN}

Como adultos jóvenes, es relevante que más de la mitad reconozca en sí mismo factores de riesgo para el ACV. Esto concuerda con los autores que afirman la tendencia creciente de la patología en esta franja etaria ${ }^{(5)}$. Hay por ventura, baja percepción de susceptibilidad. Algunos comportamientos perjudiciales, como el tabaquismo, son adquiridos frecuentemente en la adolescencia y es difícil abandonarlos. De hecho, los fumadores poseen de 2 a 4 veces mayor riesgo de padecer ACV, a lo largo de su vida, que los no fumadores o los exfumadores desde hace más de 10 años ${ }^{(21)}$. 
La hipertensión, como segundo factor de riesgo y el más referido por los hombres, era algo esperable, concordando con estudios anteriores ${ }^{(2,8)}$. Los datos son concordantes con los reportes de la DGS referentes a la región del Alentejo ${ }^{(8)}$. No menos importante como factor de riesgo, es la hipercolesterolemia, con mayor tendencia en el sexo femenino, confirmando los datos locales ${ }^{(22)}$. Los resultados anteriormente descritos sugieren que el riesgo en los participantes es elevado ya que los factores son prevalentes cerca de los 53-60 años ${ }^{(17)}$. La similitud entre los perfiles de los participantes en relación a los datos de otros estudios, apunta a la probabilidad de un riesgo incrementado en los jóvenes adultos locales. Así como en otros estudios, las variaciones regionales de riesgo deberían ser objeto de programas de intervención de carácter urgente ${ }^{(23)}$. De hecho, los riesgos no están aislados, se presentan en simultáneo, pudiendo ser agravados o potencializados.

En controversia a los comportamientos de riesgo, el conocimiento sobre el AVC es elevado. Parece contribuir a este conocimiento, la intensidad del mensaje televisivo. La accesibilidad a la televisión posibilita la emisión-recepción de mensajes intensos favoreciendo la incautación ${ }^{(20)}$. Así mismo, a pesar del conocimiento sobre el ACV, la presencia de los comportamientos de riesgo, da indicios de sub-valorización. Estos resultados van al encuentro de estudio de revisión, donde se encuentran resultados mixtos de la eficacia de las intervenciones educativas, con variaciones según los diferentes factores ${ }^{(24)}$. La posibilidad de tratamiento del ACV es desconocida por la mayoría de los participantes, hecho indicado anteriormente ${ }^{(20)}$, pero la posibilidad de cura puede incitar, la necesidad de aprender como asistir a personas con ACV en evolución. En el estudio actual, se constata una serie de desconocimientos sobre lo que hacer, pues hay participantes que no sabrían colocar al paciente para prevenir la obstrucción de la vía aérea, no evaluarían la condición respiratoria ni llamarían al número de emergencias 112. Estos hechos predisponen a la incapacidad del paciente que se evitaría, si fuera llevado a urgencia hospitalaria para ACV. Los individuos más conocedores de la patología, por otro lado, realizarían la atención y la asistencia, pero con actitudes no recomendadas (i.e., sentar a la víctima; mandar toser). Estos aspectos, incrementados por llamar al número de teléfono incorrecto, redundarían en ayuda ineficaz. Curiosamente, son los participantes con un nivel de conocimiento intermedio que actuarían más correctamente en la postura, ventilación y contacto telefónico ${ }^{(25)}$.

Los resultados sugieren que es importante y urgente la información. Revelan que el conocimiento de la actuación segura debe ser enseñado. Además, el entrenamiento de los adultos es urgente, por el impacto que tiene en la mortalidad y en la morbilidad asociada al ACV. En realidad, los programas de educación sobre señales y síntomas del ACV y actuación prehospitalaria son eficaces. Importa el reconocimiento rápido, como acudir rápido al hospital con adecuada manipulación del paciente ${ }^{(2,26)}$.

Algunas limitaciones del estudio actual se deben al tipo de muestra sin posibilidad de generalizar los resultados. El único momento, de recogida de datos, impide el seguimiento de los participantes. La utilización de medidas estandarizadas es también una fragilidad del estudio actual, reconocida en revisión de literatura ${ }^{(24,27)}$.

Sugerencias:. Sería útil la realización de estudios más grandes sobre los factores de riesgo de las patologías cardio-cerebro-vasculares. El desarrollo de un programa educativo, con valoraciones consecutivas antes y después, que permitiesen la adquisición de conocimientos y entrenamiento de competencias. El establecimiento 
de asociaciones entre la Escuela y las unidades de salud poden concretar estas ideas en proyectos útiles para la comunidad.

\section{CONCLUSIÓN}

De estos resultados, se deduce que se observa una brecha entre los comportamientos de preservación de la salud y el nivel de conocimientos sobre el ACV. Se revelan dos grandes necesidades en la población, una sobre la necesidad de concienciar para el equilibrio entre comportamientos y riesgos y otra en la capacidad para actuar con seguridad y eficacia ante una persona con ACV en curso. Pacientes y profesionales de la salud pueden contribuir con estrategias efectivas para promover comportamientos saludables Días conmemorativos o las ferias de salud son ejemplos de eventos donde se puede discutir el comportamiento para mejorar la gestión de la calidad de vida

\section{REFERENCIAS}

1. WHO. The top 10 causes of death 2010-2016 Geneve: World Health Organization; 2018 [Available from: http://www.who.int/en/news-room/factsheets/detail/the-top-10-causes-of-death.

2. Cometto MC. Manejo de enfermeira en el acidente cérebro vascular inicial. Enfermería Global [Internet]. 2005; 7:[16 p.]. Available from: https://revistas.um.es/eglobal/article/view/479/463.

3. Smajlović D. Strokes in young adults: epidemiology and prevention. Vascular Health and Risk Management. 2015;11:157-64.

4. Singhal AB, Biller J, Elkind MS, Fullerton HJ, Jauch EC, Kittner SJ, et al. Recognition and management of stroke in young adults and adolescents. Neurology. 2013;81(12):1089-97.

5. Ekker MS, Boot EM, Singhal AB, Tan KS, Debette S, Tuladhar AM, et al. Epidemiology, aetiology, and management of ischaemic stroke in young adults. Lancet Neurol. 2018;17(9):790-801.

6. Benjamin EJ, Virani SS, Callaway CW, Chamberlain AM, Chang AR, Cheng S, et al. Heart Disease and Stroke Statistics-2018 Update: A Report From the American Heart Association. Circulation. 2018;137(12):e67-e492.

7. WHO. Global status report on noncommunicable diseases 2014. Geneva: World Health Organization; 2014. 302 p.

8. Macedo M, Ferreira R. A hipertensão arterial nos cuidados de saúde primarios em Portugal: contributo para o conhecimento epidemiológico da população em 2013. Revista Factores de Risco. 2015;36:47-56.

9. Cabral NL, Longo A, Moro C, Ferst P, Oliveira FA, Vieira CV, et al. Education level explains differences in stroke incidence among city districts in Joinville, Brazil: a three-year population-based study. Neuroepidemiology. 2011;36(4):258-64.

10. Ahacic K, Trygged S, Kåreholt I. Income and education as predictors of stroke mortality after the survival of a first stroke. Stroke Res Treat. 2012;2012:983145.

11. DGS. Portugal. Prevenção e controlo do tabagismo em números - 2013. Lisboa: Direção Geral de Saúde; 2013. 152 p.

12. Ministerio da Saúde. Caraterísticas sociodemograficas dos fumadores diários em Portugal Continental: análise comparativa dos Inquéritos Nacionais de Saúde. Lisboa: Instituto Nacional de Saúde Doutor Ricardo Jorge; 2017. 
13. Macedo MEd, Lima MJ, Silva AO, Alcântara P, Ramalhinho V, Carmona J. Prevalência, Conhecimento, Tratamento e Controlo da Hipertensão em Portugal. Estudo PAP [2]. Rev Port Cardiol [Internet]. 200707 oct 2018; (26):[21-39 pp.]. Available

from: https://s3.amazonaws.com/academia.edu.documents/44934125/787.pdf?AWSAccess Keyld=AKIAIWOWYYGZ2Y53UL3A\&Expires $=1538907305 \&$ Signature $=$ ceTB8NcgpfD Xp9cVVy8FkIW\%2BCkE\%3D\&response-contentdisposition=inline \%3B\%20filename\%3DPrevalencia conhecimento tratamento e co. pdf.

14. Sousa-Uva M, Dias CM. Prevalência de Acidente Vascular Cerebral na população portuguesa: dados da amostra ECOS 2013. Boletim Epidemiológico [Internet]. $2014 \quad 07$ Oct 2018; (4):[12.4 p.]. Available from: http://repositorio.insa.pt/bitstream/10400.18/2341/3/Boletim Epidemiologico Observac oes 92014 artigo4.pdf.

15. DGS. Programa Nacional para a Diabetes 2017. Saúde DGd, editor. Lisboa2017. 20 p.

16. Ministerio da Saúde. Retrato da Saúde - Portugal. Lisboa: Serviço Nacional de Saúde; 2018.

17. Metias MM, Eisenberg N, Clemente MD, Wooster EM, Dueck AD, Wooster DL, et al. Public health campaigns and their effect on stroke knowledge in a high-risk urban population: A five-year study. Vascular. 2017:1708538117691879.

18. Almeida L, Freire T. Metodologia da Investigação em Psicologia e Educação. 5a ed. Lisboa: Psiquilíbrios; 2017.

19. Coelho R, Freitas W, Campos G, Teixeira R. Stroke awareness among cardiovascular disease patients. Arquivos de Neuro-Psiquiatria. 2008;66:209-12.

20. Bule MJ, Sim-Sim M, Correia IMTB, Falé MJM. Population's Knowledge about cerebrovascular accident (STROKE) - passers of the square Giraldo in Évora. Journal of Nursing UFPE on line [Internet]. 2016; 10(1):[65-72 pp.]. Available from: http://www.revista.ufpe.br/revistaenfermagem/index.php/revista/article/view/7709.

21. Shah RS, Cole JW. Smoking and stroke: the more you smoke the more you stroke. Expert review of cardiovascular therapy. 2010;8(7):917-32.

22. Ferreira R, Neves R. Portugal - Doenças Cérebro-Cardiovasculares em números - 2015. Lisboa: Direção Geral de Saúde; 2016.

23. O'Donnell MJ, Chin SL, Rangarajan S, Xavier D, Liu L, Zhang H, et al. Global and regional eff ects of potentially modifi able risk factors associated with acute stroke in 32 countries (INTERSTROKE): a case-control study. The Lancet [Internet]. 201608 july 2017; 388(10046):[761-75 pp.].

24. Bridgwood B, Lager KE, Mistri AK, Khunti K, Wilson AD, Modi P. Interventions for improving modifiable risk factor control in the secondary prevention of stroke. Cochrane Database Syst Rev. 2018;5:Cd009103.

25. SNS-INEM. Gestos que salvam- Posição lateral de segurança: SNS; 2017 [Available from: http://www.inem.pt/category/cidadaos/gestos-que-salvam/.

26. Yang L, Zhao Q, Zhu X, Shen X, Zhu Y, Yang L, et al. Effect of a comprehensive health education program on pre- hospital delay intentions in high-risk stroke population and caregivers. Quality of Life Research [Internet]. 201709 july 2017. Available from: http://link.springer.com.ololo.scihub.cc/article/10.1007\%2Fs11136-017-1550-4.

27. Lager KE, Mistri AK, Khunti K, Haunton VJ, Sett AK, Wilson AD. Interventions for improving modifiable risk factor control in the secondary prevention of stroke. Cochrane Database Syst Rev. 2014(5):Cd009103. 
